\title{
FILTER REGULAR SEQUENCES AND GENERALIZED LOCAL COHOMOLOGY MODULES
}

\author{
ALI FATHI, ABOLFAZL TEHRANIAN, AND HOSSEIN ZAKERI
}

\begin{abstract}
Let $\mathfrak{a}, \mathfrak{b}$ be ideals of a commutative Noetherian $\operatorname{ring} R$ and let $M$, $N$ be finite $R$-modules. The concept of an $\mathfrak{a}$-filter grade of $\mathfrak{b}$ on $M$ is introduced and several characterizations and properties of this notion are given. Then, using the above characterizations, we obtain some results on generalized local cohomology modules $\mathrm{H}_{\mathfrak{a}}^{i}(M, N)$. In particular, first we determine the least integer $i$ for which $\mathrm{H}_{\mathfrak{a}}^{i}(M, N)$ is not Artinian. Then we prove that $\mathrm{H}_{\mathfrak{a}}^{i}(M, N)$ is Artinian for all $i \in \mathbb{N}_{0}$ if and only if $\operatorname{dim} R /(\mathfrak{a}+\operatorname{Ann} M+\operatorname{Ann} N)=0$. Also, we establish the Nagel-Schenzel formula for generalized local cohomology modules. Finally, in a certain case, the set of attached primes of $\mathrm{H}_{\mathfrak{a}}^{i}(M, N)$ is determined and a comparison between this set and the set of attached primes of $\mathrm{H}_{\mathfrak{a}}^{i}(N)$ is given.
\end{abstract}

\section{INTRODUCTION}

Throughout this paper, $R$ is a commutative Noetherian ring with nonzero identity, $\mathfrak{a}, \mathfrak{b}$ are ideals of $R$ and $M, N, L$ are finite $R$-modules. We will use $\mathbb{N}$ (respectively $\mathbb{N}_{0}$ ) to denote the set of positive (respectively non-negative) integers. The generalized local cohomology functor was first introduced, in the local case, by Herzog [10] and, in the general case, by Bijan-Zadeh [3]. The $i$-th generalized local cohomology functor $\mathrm{H}_{\mathfrak{a}}^{i}(\cdot, \cdot)$ is defined by

$$
\mathrm{H}_{\mathfrak{a}}^{i}(X, Y)=\underset{n}{\lim } \operatorname{Ext}_{R}^{i}\left(X / \mathfrak{a}^{n} X, Y\right)
$$

for all $R$-modules $X, Y$ and $i \in \mathbb{N}_{0}$. Clearly, this notion is a natural generalization of the ordinary local cohomology functor [5].

There is a lot of current interest in the theory of filter regular sequences in commutative algebra; and, in recent years, there have appeared many papers concerned with the role of these sequences in the theory of local cohomology. In particular case, when one works on a local ring, the concept of a filter regular sequence has been studied in [23, 26] and has led to some interesting results. We will denote the supremum of all numbers $n \in \mathbb{N}_{0}$ for which there exists an $\mathfrak{a}$-filter regular $M$ sequence of length $n$ in $\mathfrak{b}$ by $f-\operatorname{grad}(\mathfrak{a}, \mathfrak{b}, M)$. In a local ring $(R, \mathfrak{m}), f-\operatorname{grad}(\mathfrak{m}, \mathfrak{a}, M)$ is known as $\mathrm{f}-\operatorname{depth}(\mathfrak{a}, M)$. Lü and Tang [13] proved that

$$
\text { f-depth }(\mathfrak{a}, M)=\inf \left\{i \in \mathbb{N}_{0} \mid \operatorname{dim} \operatorname{Ext}_{R}^{i}(R / \mathfrak{a}, M)>0\right\}
$$

and that $\mathrm{f}-\operatorname{depth}(\mathfrak{a}, M)$ is the least integer $i$ such that $\mathrm{H}_{\mathfrak{a}}^{i}(M)$ is not Artinian. As a theorem, we generalize their results and characterize $f-\operatorname{grad}(\mathfrak{a}, \mathfrak{b}, M)$ to non local

2010 Mathematics Subject Classification. 13D45, 13E10.

Key words and phrases. generalized local cohomology module, filter regular sequence, NagelSchenzel formula, Artinianness, Attached prime. 
cases as follows.

$$
\begin{aligned}
\mathrm{f}-\operatorname{grad}(\mathfrak{a}, \mathfrak{b}, M) & =\inf \left\{i \in \mathbb{N}_{0} \mid \operatorname{Supp} \operatorname{Ext}_{R}^{i}(R / \mathfrak{b}, M) \nsubseteq V(\mathfrak{a})\right\} \\
& =\inf \left\{i \in \mathbb{N}_{0} \mid \operatorname{Supp} \mathrm{H}_{\mathfrak{b}}^{i}(M) \nsubseteq V(\mathfrak{a})\right\}, \\
\mathfrak{f}-\operatorname{grad}(\mathfrak{a}, \mathfrak{b}+\operatorname{Ann} N, M) & =\inf \left\{i \in \mathbb{N}_{0} \mid \operatorname{Supp} \mathrm{H}_{\mathfrak{b}}^{i}(N, M) \nsubseteq V(\mathfrak{a})\right\},
\end{aligned}
$$

and

$$
\begin{aligned}
\sup _{A \in \mathcal{M}} \operatorname{f}-\operatorname{grad}\left(\bigcap_{\mathfrak{m} \in A} \mathfrak{m}, \mathfrak{a}\right. & +\operatorname{Ann} M, N) \\
& =\inf \left\{i \in \mathbb{N}_{0} \mid \mathrm{H}_{\mathfrak{a}}^{i}(M, N) \text { is not Artinian }\right\} \\
& =\inf \left\{i \in \mathbb{N}_{0} \mid \operatorname{Supp}_{\mathfrak{a}}^{i}(M, N) \nsubseteq \max (R)\right\} \\
& =\inf \left\{i \in \mathbb{N}_{0} \mid \operatorname{Supp} \mathrm{H}_{\mathfrak{a}}^{i}(M, N) \nsubseteq A \text { for all } A \in \mathcal{M}\right\} \\
& =\inf \left\{i \in \mathbb{N}_{0} \mid \operatorname{dim} \operatorname{Ext}_{R}^{i}(M / \mathfrak{a} M, N)>0\right\}
\end{aligned}
$$

where $\mathcal{M}$ is the set of all finite subsets of $\max (R)$.

As an application of this theorem, we show that, if $n \in \mathbb{N}$, then $\mathrm{H}_{\mathfrak{a}}^{i}(M, N)$ is Artinian for all $i<n$ if and only if $\mathrm{H}_{\mathfrak{a} R_{\mathfrak{p}}}^{i}\left(M_{\mathfrak{p}}, N_{\mathfrak{p}}\right)$ is Artinian for all $i<n$ and all prime ideals $\mathfrak{p}$. Also, we prove that $\mathrm{H}_{\mathfrak{a}}^{i}(M, N)$ is an Artinian $R$-module for all $i \in \mathbb{N}_{0}$ if and only if $\operatorname{dim} R /(\mathfrak{a}+\operatorname{Ann} M+\operatorname{Ann} N)=0$. In particular, $\operatorname{Ext}_{R}^{i}(M, N)$ has finite length for all $i \in \mathbb{N}_{0}$ if and only if $\operatorname{dim} R /(\operatorname{Ann} M+\operatorname{Ann} N)=0$.

Let $x_{1}, \ldots, x_{n}$ be an $\mathfrak{a}$-filter regular $N$-sequence in $\mathfrak{a}$. Then the formula

$$
\mathrm{H}_{\mathfrak{a}}^{i}(N)= \begin{cases}\mathrm{H}_{\left(x_{1}, \ldots, x_{n}\right)}^{i}(N) & \text { if } i<n, \\ \mathrm{H}_{\mathfrak{a}}^{i-n}\left(\mathrm{H}_{\left(x_{1}, \ldots, x_{n}\right)}^{n}(N)\right) & \text { if } i \geq n,\end{cases}
$$

is known as Nagel-Schenzel formula (see [20 and [11). We generalize the above formula for the generalized local cohomology modules. Indeed, we prove that:

(i) $\mathrm{H}_{\mathfrak{a}}^{i}(M, N) \cong \mathrm{H}_{\left(x_{1}, \ldots, x_{n}\right)}^{i}(M, N)$ for all $i<n$;

(ii) if $\operatorname{proj} \operatorname{dim} M=d$ and $L$ is projective, then

$$
\mathrm{H}_{\mathfrak{a}}^{i+n}\left(M \otimes_{R} L, N\right) \cong \mathrm{H}_{\mathfrak{a}}^{i}\left(M, \mathrm{H}_{\left(x_{1}, \ldots, x_{n}\right)}^{n}(L, N)\right)
$$

for all $i \geq d$.

Assume that $\bar{R}=R /(\mathfrak{a}+\operatorname{Ann} M+\operatorname{Ann} N)$ and that the ideal $\mathfrak{r}$ is the inverse image of the Jacobson radical of $\bar{R}$ in $R$. If $\bar{R}$ is semi local, then, by using the isomorphisms described in (i) and Theorem 4.2, we prove that

$$
\begin{aligned}
\mathrm{f}-\operatorname{grad}(\mathfrak{r}, \mathfrak{a}+\operatorname{Ann} M, N) & =\inf \left\{i \in \mathbb{N}_{0} \mid \mathrm{H}_{\mathfrak{a}}^{i}(M, N) \text { is not Artinian }\right\} \\
& =\inf \left\{i \in \mathbb{N}_{0} \mid \mathrm{H}_{\mathfrak{a}}^{i}(M, N) \varsubsetneqq \mathrm{H}_{\mathfrak{r}}^{i}(M, N)\right\} .
\end{aligned}
$$

Let $(R, \mathfrak{m})$ be a local ring and $\operatorname{dim} N=n$. Macdonald and Sharp [15, Theorem 2.2 ] show that

$$
\operatorname{Att}_{\mathfrak{m}}^{n}(N)=\{\mathfrak{p} \in \operatorname{Ass} N \mid \operatorname{dim} R / \mathfrak{p}=n\} .
$$

As an extension of this result, Dibaei and Yassemi [8, Theorem A] proved

$$
\operatorname{Att}_{\mathfrak{a}}^{n}(N)=\left\{\mathfrak{p} \in \operatorname{Ass} N \mid \operatorname{cd}_{\mathfrak{a}}(R / \mathfrak{p})=n\right\},
$$


where $\operatorname{cd}_{\mathfrak{a}}(M)$ is the greatest integer $i$ such that $\mathrm{H}_{\mathfrak{a}}^{i}(M) \neq 0$. Finally, if $d=$ $\operatorname{proj} \operatorname{dim} M<\infty$, then $\mathrm{Gu}$ and Chu [9, Theorem 2.3] proved that $\mathrm{H}_{\mathfrak{a}}^{n+d}(M, N)$ is Artinian and

$$
\operatorname{Att}_{\mathfrak{a}}^{n+d}(M, N)=\left\{\mathfrak{p} \in \operatorname{Ass} N \mid \operatorname{cd}_{\mathfrak{a}}(M, R / \mathfrak{p})=n+d\right\},
$$

where, for an $R$-module $Y, \operatorname{cd}_{\mathfrak{a}}(M, Y)$ is the greatest integer $i$ such that $\mathrm{H}_{\mathfrak{a}}^{i}(M, Y) \neq$ 0 . Notice that $\operatorname{cd}_{\mathfrak{a}}(M, N) \leq d+n$ [3, Lemma 5.1]. We prove the above result in general case where $R$ is not necessarily local. As a corollary we deduce that

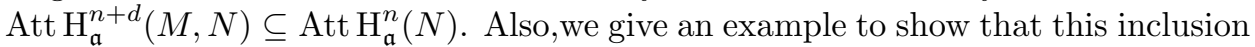
may be strict. Indeed, our example not only show that the Theorem 2.1 of [17] is not true, but it also rejects all of the following conclusions in [17.

Finally, Let $\operatorname{proj} \operatorname{dim} M=d<\infty$ and $\operatorname{dim} N=n<\infty$ and $\mathfrak{b}=\operatorname{Ann} \mathrm{H}_{\mathfrak{a}}^{n}(N)$. We prove that, if $R / \mathfrak{b}$ is a complete semilocal ring, then

$$
\operatorname{Att}_{\mathfrak{a}}^{n+d}(M, N)=\operatorname{Supp} \operatorname{Ext}_{R}^{d}(M, R) \cap \operatorname{Att}_{\mathfrak{a}}^{n}(N) .
$$

In particular, if in addition, $\operatorname{proj} \operatorname{dim}_{R_{\mathfrak{p}}} M_{\mathfrak{p}}=\operatorname{proj} \operatorname{dim} M$ for all $\mathfrak{p} \in \operatorname{Supp} M$, then

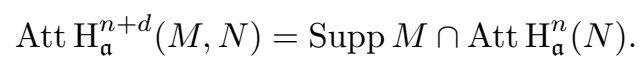

\section{Filter Regular Sequences}

We say that a sequence $x_{1}, \ldots, x_{n}$ of elements of $R$ is an $\mathfrak{a}$-filter regular $M$ sequence, if $x_{i} \notin \mathfrak{p}$ for all $\mathfrak{p} \in$ Ass $M /\left(x_{1}, \ldots, x_{i-1}\right) M \backslash V(\mathfrak{a})$ and for all $i=1, \ldots, n$. In addition, if $x_{1}, \ldots, x_{n}$ belong to $\mathfrak{b}$, then we say that $x_{1}, \ldots, x_{n}$ is an $\mathfrak{a}$-filter regular $M$-sequence in $\mathfrak{b}$. Note that $x_{1}, \ldots, x_{n}$ is an $R$-filter regular $M$-sequence if and only if it is a weak $M$-sequence in the sense of [6, Definition 1.1.1].

Some parts of the next elementary proposition are included in [20, Proposition $2.2]$ in the case where $(R, \mathfrak{m})$ is local and $\mathfrak{a}=\mathfrak{m}$.

Proposition 2.1. Let $x_{1}, \ldots, x_{n}$ be a sequence of elements of $R$ and $n \in \mathbb{N}$. Then the following statements are equivalent.

(i) $x_{1}, \ldots, x_{n}$ is an $\mathfrak{a}$-filter regular $M$-sequence.

(ii) $\operatorname{Supp}\left(\left(x_{1}, \ldots, x_{i-1}\right) M:_{M} x_{i}\right) /\left(x_{1}, \ldots, x_{i-1}\right) M \subseteq V$ (a) for all $i=1, \ldots, n$.

(iii) $x_{1} / 1, \ldots, x_{n} / 1$ is a weak $M_{\mathfrak{p}}$-sequence for all $\mathfrak{p} \in \operatorname{Supp} M \backslash V(\mathfrak{a})$.

(iv) $x_{1}^{\alpha_{1}}, \ldots, x_{n}^{\alpha_{n}}$ is an $\mathfrak{a}$-filter regular $M$-sequence for all positive integers $\alpha_{1}, \ldots, \alpha_{n}$.

(v) $x_{i}$ is a weak $\left(M /\left(x_{1}, \ldots, x_{i-1}\right) M\right) / \Gamma_{\mathfrak{a}}\left(M /\left(x_{1}, \ldots, x_{i-1}\right) M\right)$-sequence for all $i=1, \ldots, n$.

(vi) $\left(x_{1}, \ldots, x_{i-1}\right) M:_{M} x_{i} \subseteq\left(x_{1}, \ldots, x_{i-1}\right) M:_{M}\langle\mathfrak{a}\rangle$ for all $i=1, \ldots, n$, where $N: M\langle\mathfrak{a}\rangle=\left\{x \in M \mid \mathfrak{a}^{m} x \subseteq N\right.$ for some $\left.m \in \mathbb{N}\right\}$ for any submodule $N$ of $M$.

It is clear from definition, that, for a given $n \in \mathbb{N}$, one can find an $\mathfrak{a}$-filter regular $M$-sequence of length $n$. The following theorem characterizes the existence of an $\mathfrak{a}$-filter regular $M$-sequence of length $n$ in $\mathfrak{b}$.

Theorem 2.2. Let $n \in \mathbb{N}$. Then the following statements are equivalent.

(i) $\mathfrak{b}$ contains an $\mathfrak{a}$-filter regular $M$-sequence of length $n$.

(ii) Any $\mathfrak{a}$-filter regular $M$-sequence in $\mathfrak{b}$ of length less than $n$ can be extended to an $\mathfrak{a}$-filter regular $M$-sequence of length $n$ in $\mathfrak{b}$.

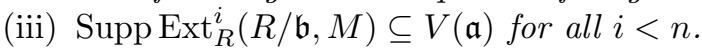

(iv) If $\operatorname{Supp} N=V(\mathfrak{b})$, then $\operatorname{Supp} \operatorname{Ext}_{R}^{i}(N, M) \subseteq V(\mathfrak{a})$ for all $i<n$.

(v) $\operatorname{Supp}_{\mathfrak{b}}^{i}(M) \subseteq V(\mathfrak{a})$ for all $i<n$. 
(vi) If Ann $N \subseteq \mathfrak{b}$, then $\operatorname{Supp}_{\mathfrak{b}}^{i}(N, M) \subseteq V$ (a) for all $i<n$.

Proof. The implications (ii) $\Rightarrow$ (i), (iv) $\Rightarrow$ (iii) and (vi) $\Rightarrow$ (v) are clear.

(i) $\Rightarrow$ (ii). Assume the contrary that $x_{1}, \ldots, x_{t}$ is an $\mathfrak{a}$-filter regular $M$-sequence in $\mathfrak{b}$ such that $t<n$ and that it can not be extended to an $\mathfrak{a}$-filter regular $M$-sequence of length $n$ in $\mathfrak{b}$. Then $\mathfrak{b} \subseteq \mathfrak{p}$ for some $\mathfrak{p} \in \operatorname{Ass} M /\left(x_{1}, \ldots, x_{t}\right) M \backslash V(\mathfrak{a})$. So that $\mathfrak{b} R_{\mathfrak{p}} \subseteq \mathfrak{p} R_{\mathfrak{p}} \in \operatorname{Ass}_{R_{\mathfrak{p}}} M_{\mathfrak{p}} /\left(x_{1} / 1, \ldots, x_{t} / 1\right) M_{\mathfrak{p}}$. It follows that $x_{1} / 1, \ldots, x_{t} / 1$ is a maximal $M_{\mathfrak{p}}$-sequence in $\mathfrak{b} R_{\mathfrak{p}}$, which is a contradiction in view of the hypothesis, Proposition 2.1 and [6, Theorem 1.2.5].

(i) $\Rightarrow$ (iv) Suppose that $x_{1}, \ldots, x_{n}$ is an $\mathfrak{a}$-filter regular $M$-sequence in $\mathfrak{b}$. Let $t \in \mathbb{N}$ be such that $x_{i}^{t} \in \operatorname{Ann} N$ for all $i=1, \ldots, n$. By Proposition 2.1, for any $\mathfrak{p} \in \operatorname{Supp} M \backslash V(\mathfrak{a}), x_{1}^{t} / 1, \ldots, x_{n}^{t} / 1$ is a weak $M_{\mathfrak{p}}$-sequence in $\operatorname{Ann}_{R_{\mathfrak{p}}} N_{\mathfrak{p}}$. So that, for all $i<n$, we have $\operatorname{Ext}_{R_{\mathfrak{p}}}^{i}\left(N_{\mathfrak{p}}, M_{\mathfrak{p}}\right)=0$. Therefore (iv) holds.

(i) $\Rightarrow$ (vi) Suppose that $x_{1}, \ldots, x_{n}$ is an $\mathfrak{a}$-filter regular $M$-sequence in $\mathfrak{b}$. For any $\mathfrak{p} \in \operatorname{Supp} M \backslash V(\mathfrak{a}), x_{1} / 1, \ldots, x_{n} / 1$ is a weak $M_{\mathfrak{p}}$-sequence in $\mathfrak{b} R_{\mathfrak{p}}$. So that, by [3, Proposition 5.5], $\mathrm{H}_{\mathfrak{b} R_{\mathfrak{p}}}^{i}\left(N_{\mathfrak{p}}, M_{\mathfrak{p}}\right)=0$ for all $i<n$. This proves the implication (i) $\Rightarrow$ (vi).

Next we prove the implications (iii) $\Rightarrow(\mathrm{i})$ and $(\mathrm{v}) \Rightarrow(\mathrm{i})$ by induction on $n$. Let $n=1$. In either cases $\operatorname{Supp} \operatorname{Hom}_{R}(R / \mathfrak{b}, M) \subseteq V(\mathfrak{a})$. Therefore (i) holds. Suppose that, for all $i \in \mathbb{N}_{0}, T^{i}(\cdot)$ is either $\operatorname{Ext}_{R}^{i}(R / \mathfrak{b}, \cdot)$ or $\mathrm{H}_{\mathfrak{b}}^{i}(\cdot)$. Assume that $n>1$ and $\operatorname{Supp} T^{i}(M) \subseteq V(\mathfrak{a})$ for all $i<n$. Then $\mathfrak{b}$ contains an $\mathfrak{a}$-filter regular $M$-sequence, say $x_{1}$. The exact sequences

$$
0 \longrightarrow 0::_{M} x_{1} \longrightarrow M \stackrel{x_{1}}{\longrightarrow} x_{1} M \longrightarrow 0
$$

and

$$
0 \longrightarrow x_{1} M \longrightarrow M \longrightarrow M / x_{1} M \longrightarrow 0
$$

induce the long exact sequences

$$
\cdots \longrightarrow T^{i}\left(0:_{M} x_{1}\right) \longrightarrow T^{i}(M) \longrightarrow T^{i}\left(x_{1} M\right) \longrightarrow T^{i+1}\left(0:_{M} x_{1}\right) \longrightarrow \cdots
$$

and

$$
\cdots \longrightarrow T^{i}\left(x_{1} M\right) \longrightarrow T^{i}(M) \longrightarrow T^{i}\left(M / x_{1} M\right) \longrightarrow T^{i+1}\left(x_{1} M\right) \longrightarrow \cdots .
$$

Since Supp $0:_{M} x_{1} \subseteq V(\mathfrak{a})$, by Proposition 2.1, it follows that Supp $T^{i}\left(0:_{M} x_{1}\right) \subseteq$ $V(\mathfrak{a})$ for all $i \in \mathbb{N}_{0}$. Therefore, using the above long exact sequences, we have $\operatorname{Supp} T^{i}\left(M / x_{1} M\right) \subseteq V(\mathfrak{a})$ for all $i<n-1$. Hence, by inductive hypothesis, $\mathfrak{b}$ contains an a-filter regular $M / x_{1} M$-sequence of length $n-1$ such as $x_{2}, \ldots, x_{n}$. This completes the inductive step, since $x_{1}, \ldots, x_{n}$ is an $\mathfrak{a}$-filter regular $M$-sequence in $\mathfrak{b}$.

Remark 2.3. One may use Theorem 2.2 (iii) $\Rightarrow$ (ii) and Proposition 2.1 to see that Supp $M / \mathfrak{b} M \subseteq V(\mathfrak{a})$ if and only if, for each $n \in \mathbb{N}$, there exists an $\mathfrak{a}$-filter regular $M$-sequence of length $n$ in $\mathfrak{b}$. Moreover, if Supp $M / \mathfrak{b} M \nsubseteq V(\mathfrak{a})$, then it follows from Theorem 2.2 that any two maximal $\mathfrak{a}$-filter regular $M$-sequences in $\mathfrak{b}$ have the same length. Therefore, we may give the following.

Definition 1. Let $\operatorname{Supp} M / \mathfrak{b} M \nsubseteq V(\mathfrak{a})$. Then the common length of all maximal $\mathfrak{a}$-filter regular $M$-sequences in $\mathfrak{b}$ is denoted by $f-\operatorname{grad}(\mathfrak{a}, \mathfrak{b}, M)$ and is called the $\mathfrak{a}$ filter grade of $\mathfrak{b}$ on $M$. We set $f-\operatorname{grad}(\mathfrak{a}, \mathfrak{b}, M)=\infty$ whenever $\operatorname{Supp} M / \mathfrak{b} M \subseteq V(\mathfrak{a})$. 
Let $(R, \mathfrak{m})$ be a local ring. Then the $\mathfrak{m}$-filter grade of $\mathfrak{b}$ on $M$ is called the filter depth of $\mathfrak{b}$ on $M$ and is denoted by $\mathrm{f}-\operatorname{depth}(\mathfrak{b}, M)$. Notice that, by Remark 2.3 $\mathrm{f}$-depth $(\mathfrak{b}, M)<\infty$ if and only if $M / \mathfrak{b} M$ has finite length.

Remark 2.4. The following equalities follows immediately from Theorem 2.2

$$
\begin{aligned}
\mathrm{f}-\operatorname{grad}(\mathfrak{a}, \operatorname{Ann} N, M) & =\inf \left\{i \in \mathbb{N}_{0} \mid \operatorname{Supp} \operatorname{Ext}_{R}^{i}(N, M) \nsubseteq V(\mathfrak{a})\right\}, \\
\mathrm{f}-\operatorname{grad}(\mathfrak{a}, \mathfrak{b}+\operatorname{Ann} N, M) & =\inf \left\{i \in \mathbb{N}_{0} \mid \operatorname{Supp~}_{\mathfrak{b}}^{i}(N, M) \nsubseteq V(\mathfrak{a})\right\} .
\end{aligned}
$$

In particular,

$$
\begin{aligned}
\mathrm{f}-\operatorname{grad}(\mathfrak{a}, \mathfrak{b}, M) & =\inf \left\{i \in \mathbb{N}_{0} \mid \operatorname{Supp} \operatorname{Ext}_{R}^{i}(R / \mathfrak{b}, M) \nsubseteq V(\mathfrak{a})\right\} \\
& =\inf \left\{i \in \mathbb{N}_{0} \mid \operatorname{Supp~}_{\mathfrak{b}}^{i}(M) \nsubseteq V(\mathfrak{a})\right\} .
\end{aligned}
$$

Suppose in addition that $(R, \mathfrak{m})$ is local. Then

$$
\begin{aligned}
\mathrm{f}-\operatorname{depth}(\mathfrak{b}, M) & =\inf \left\{i \in \mathbb{N}_{0} \mid \operatorname{dim} \operatorname{Ext}_{R}^{i}(R / \mathfrak{b}, M)>0\right\} \\
& =\inf \left\{i \in \mathbb{N}_{0} \mid \operatorname{Supp~}_{\mathfrak{b}}^{i}(M) \nsubseteq\{\mathfrak{m}\}\right\} .
\end{aligned}
$$

\section{A generalization of Nagel-Schenzel Formula}

Let $x_{1}, \ldots, x_{n}$ be an $\mathfrak{a}$-filter regular $M$-sequence in $\mathfrak{a}$. Then, by [11, Proposition $1.2]$,

$$
\mathrm{H}_{\mathfrak{a}}^{i}(M)= \begin{cases}\mathrm{H}_{\left(x_{1}, \ldots, x_{n}\right)}^{i}(M) & \text { if } i<n, \\ \mathrm{H}_{\mathfrak{a}}^{i-n}\left(\mathrm{H}_{\left(x_{1}, \ldots, x_{n}\right)}^{n}(M)\right) & \text { if } i \geq n .\end{cases}
$$

This formula was first obtained by Nagel and Schenzel, in [20, Lemma 3.4], in the case where $(R, \mathfrak{m})$ is a local ring and $\mathfrak{a}=\mathfrak{m}$. Afterwards Khashyarmanesh, Yassi and Abbasi [12, Theorem 3.2] and Mafi [16, Lemma 2.8] generalized the second part of this formula for the generalized local cohomology modules as follows.

Suppose that $M$ has finite projective dimension $d$ and that $x_{1}, \ldots, x_{n}$ is an $\mathfrak{a}$ filter regular $N$-sequence in $\mathfrak{a}$. Then

$$
\mathrm{H}_{\mathfrak{a}}^{i+n}(M, N) \cong \mathrm{H}_{\mathfrak{a}}^{i}\left(M, \mathrm{H}_{\left(x_{1}, \ldots, x_{n}\right)}^{n}(N)\right)
$$

for all $i \geq d$.

The following Theorem establishes the Nagel-Schenzel formula for the generalized local cohomology modules. The first part of the following theorem is needed in the proof of the Corollary 4.5 .

Theorem 3.1. Let $x_{1}, \ldots, x_{n}$ be an $\mathfrak{a}$-filter regular $N$-sequence in $\mathfrak{a}$. Then the following statements hold.

(i) $\mathrm{H}_{\mathfrak{a}}^{i}(M, N) \cong \mathrm{H}_{\left(x_{1}, \ldots, x_{n}\right)}^{i}(M, N)$ for all $i<n$.

(ii) If $\operatorname{proj} \operatorname{dim} M=d<\infty$ and $L$ is projective, then

$$
\mathrm{H}_{\mathfrak{a}}^{i+n}\left(M \otimes_{R} L, N\right) \cong \mathrm{H}_{\mathfrak{a}}^{i}\left(M, \mathrm{H}_{\left(x_{1}, \ldots, x_{n}\right)}^{n}(L, N)\right)
$$

for all $i \geq d$.

Proof. (i) Set $\boldsymbol{x}=x_{1}, \ldots, x_{n}$. Since $\Gamma_{\mathfrak{a}}(N) \subseteq \Gamma_{(\boldsymbol{x})}(N)$ we have a natural monomorphism $\varphi_{M, N}: \mathrm{H}_{\mathfrak{a}}^{0}(M, N) \rightarrow \mathrm{H}_{(\boldsymbol{x})}^{0}(M, N)$. Now, let $\mu_{i}(\mathfrak{p}, N)$ be the $i$-th Bass number

of $N$ with respect to a prime ideal $\mathfrak{p}$ and let $0 \longrightarrow E^{0} \stackrel{d^{0}}{\longrightarrow} E^{1} \stackrel{d^{1}}{\longrightarrow} E^{2} \longrightarrow \cdots$ be 
the minimal injective resolution of $N$. Then, by Proposition 2.1. $\mu_{i}(\mathfrak{p}, N)=0$ for all $\mathfrak{p} \in \operatorname{Supp} N \cap V(\boldsymbol{x}) \backslash V(\mathfrak{a})$ and all $i<n$. So

$$
\begin{aligned}
\Gamma_{\mathfrak{a}}\left(E^{i}\right) & =\bigoplus_{\mathfrak{p} \in \operatorname{Supp} N \cap V(\mathfrak{a})} E(R / \mathfrak{p})^{\mu_{i}(\mathfrak{p}, N)} \\
& =\bigoplus_{\mathfrak{p} \in \operatorname{Supp} N \cap V(\boldsymbol{x})} E(R / \mathfrak{p})^{\mu_{i}(\mathfrak{p}, N)}=\Gamma_{(\boldsymbol{x})}\left(E^{i}\right)
\end{aligned}
$$

for all $i<n$. Therefore $\varphi_{M, E^{i}}$ is an isomorphism for all $i<n$. Now let $i<n$. Since $\varphi_{M, E^{i-1}}$ and $\varphi_{M, E^{i}}$ are isomorphisms and $\varphi_{M, E^{i+1}}$ is a monomorphism, one can use the following commutative diagram

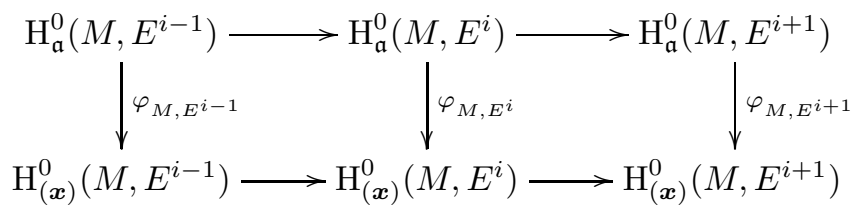

to see that the induced homomorphism

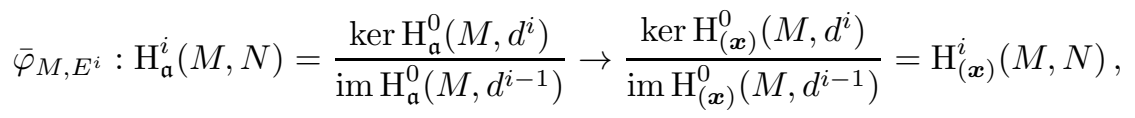

is an isomorphism.

(ii) Set $F(\cdot)=\mathrm{H}_{\mathfrak{a}}^{0}(M, \cdot)$ and $G(\cdot)=\mathrm{H}_{(\boldsymbol{x})}^{0}(L, \cdot)$. Then $F$ and $G$ are left exact functors and $F G(\cdot) \cong \mathrm{H}_{\mathfrak{a}}^{0}\left(M \otimes_{R} L, \cdot\right)$. Furthermore if $E$ is an injective $R$-module and $\mathrm{R}^{p} F\left(p \in \mathbb{N}_{0}\right)$ is the $p$-th right derived functor of $F$, then it follows from [27, Lemma 1.1] and(i) that

$$
\begin{aligned}
\mathrm{R}^{p} F(G(E)) & =\mathrm{H}_{\mathfrak{a}}^{p}\left(M, \mathrm{H}_{(\boldsymbol{x})}^{0}(L, E)\right) \cong \mathrm{H}_{\mathfrak{a}}^{p}\left(M, \mathrm{H}_{\mathfrak{a}}^{0}(L, E)\right) \\
& \cong \operatorname{Ext}_{R}^{p}\left(M, \operatorname{Hom}_{R}\left(L, \Gamma_{\mathfrak{a}}(E)\right)\right)=0
\end{aligned}
$$

for all $p \geq 1$. This yields the following spectral sequence

$$
E_{2}^{p, q}=\mathrm{H}_{\mathfrak{a}}^{p}\left(M, \mathrm{H}_{(\boldsymbol{x})}^{q}(L, N)\right) \underset{p}{\Longrightarrow} \mathrm{H}_{\mathfrak{a}}^{p+q}\left(M \otimes_{R} L, N\right)
$$

(see for example [21, Theorem 11.38] ). Let $t=p+q \geq d+n$. If $q>n$, then $\mathrm{H}_{(\boldsymbol{x})}^{q}(N)=0$ by [5, Corollary 3.3.3]. Since $L$ is projective, it therefore follows that $\mathrm{H}_{(\boldsymbol{x})}^{q}(L, N)=0$. On the other hand if $q<n$, then $p>d=\operatorname{proj} \operatorname{dim} M$. Hence

$$
E_{2}^{p, q}=\mathrm{H}_{\mathfrak{a}}^{p}\left(M, \mathrm{H}_{(\boldsymbol{x})}^{q}(L, N)\right) \cong \mathrm{H}_{\mathfrak{a}}^{p}\left(M, \mathrm{H}_{\mathfrak{a}}^{q}(L, N)\right) \cong \operatorname{Ext}_{R}^{p}\left(M, \mathrm{H}_{\mathfrak{a}}^{q}(L, N)\right)=0 .
$$

Therefore, for $t \geq n+d$, there is a collapsing on the line $\mathfrak{q}=n$. Thus, there are isomorphisms

$$
\mathrm{H}_{\mathfrak{a}}^{t-n}\left(M, \mathrm{H}_{(\boldsymbol{x})}^{n}(L, N)\right) \cong \mathrm{H}_{\mathfrak{a}}^{t}\left(M \otimes_{R} L, N\right)
$$

for all $t \geq n+d$.

\section{Artinianness of Generalized LOCAL COHOMOLOGY MODUlES}

Let $(R, \mathfrak{m})$ be a Noetherian local ring. In view of [19, Theorem 3.1] and [13, Theorem 3.10], one can see that $\mathrm{f}-\operatorname{depth}(\mathfrak{a}, M)$ is the least integer $i$ for which $\mathrm{H}_{\mathfrak{a}}^{i}(M)$ is not Artinian. Also, as a main result, it was proved in [7, Theorem 2.2] that f-depth $(\mathfrak{a}+\operatorname{Ann} M, N)$ is the least integer $i$ such that $\mathrm{H}_{\mathfrak{a}}^{i}(M, N)$ is not Artinian. 
We use rather a short argument to generalize this to the case in which $R$ is not necessarily a local ring. The following lemma is elementary.

Lemma 4.1 (22 Exercise 8.49). Let $X$ be an Artinian $R$-module, then Ass $X=$ Supp $X$ is a finite subset of $\max (R)$.

Theorem 4.2. Let $\mathcal{M}$ be the set of all finite subsets of $\max (R)$. Then

$$
\begin{aligned}
\sup _{A \in \mathcal{M}} \mathrm{f}-\operatorname{grad}\left(\cap_{\mathfrak{m} \in A} \mathfrak{m}, \mathfrak{a}\right. & +\operatorname{Ann} M, N) \\
& =\inf \left\{i \in \mathbb{N}_{0} \mid \mathrm{H}_{\mathfrak{a}}^{i}(M, N) \text { is not Artinian }\right\} \\
& =\inf \left\{i \in \mathbb{N}_{0} \mid \operatorname{Supp} \mathrm{H}_{\mathfrak{a}}^{i}(M, N) \nsubseteq \max (R)\right\} \\
& =\inf \left\{i \in \mathbb{N}_{0} \mid \operatorname{Supp} \mathrm{H}_{\mathfrak{a}}^{i}(M, N) \nsubseteq A \text { for all } A \in \mathcal{M}\right\}
\end{aligned}
$$

Proof. Since $\mathrm{H}_{\mathfrak{a}}^{i}(M, N) \cong \mathrm{H}_{\mathfrak{a}+\operatorname{Ann} M}^{i}(M, N)$, we can assume that Ann $M \subseteq \mathfrak{a}$. It is clear that

$$
\sup _{A \in \mathcal{M}} f-\operatorname{grad}\left(\cap_{\mathfrak{m} \in A} \mathfrak{m}, \mathfrak{a}, N\right)=\inf \left\{i \in \mathbb{N}_{0} \mid \operatorname{Supp~}_{\mathfrak{a}}^{i}(M, N) \nsubseteq A \text { for all } A \in \mathcal{M}\right\} .
$$

Let $\mathcal{S}$ be either $\left\{X \in \mathcal{C}_{R} \mid \operatorname{Supp} X \subseteq \max (R)\right\}$ or $\left\{X \in \mathcal{C}_{R} \mid\right.$ Supp $X \subseteq A$ for some $A \in \mathcal{M}\}$, where $\mathcal{C}_{R}$ is the category of $R$-modules. Set $r=\inf \left\{i \in \mathbb{N}_{0} \mid \mathrm{H}_{\mathfrak{a}}^{i}(M, N)\right.$ is not Artinian $\}$ and $s=\inf \left\{i \in \mathbb{N}_{0} \mid \mathrm{H}_{\mathfrak{a}}^{i}(M, N) \notin \mathcal{S}\right\}$. By Lemma 4.1, $r \leq s$. If $r=\infty$, there is noting to prove. Assume that $r<\infty$. We show by induction on $r$, that $\mathrm{H}_{\mathfrak{a}}^{r}(M, N) \notin \mathcal{S}$.

If $r=0$, then $\mathrm{H}_{\mathfrak{a}}^{0}(M, N) \notin \mathcal{S}$. Now suppose, inductively, that $r>0$ and that the result has been proved for smaller values of $r$. In view of [27, Lemma 1.1] the exact sequence

$$
0 \longrightarrow \Gamma_{\mathfrak{a}}(N) \longrightarrow N \longrightarrow N / \Gamma_{\mathfrak{a}}(N) \longrightarrow 0
$$

induces the following long exact sequence

$$
\begin{aligned}
\cdots & \longrightarrow \operatorname{Ext}_{R}^{i}\left(M, \Gamma_{\mathfrak{a}}(N)\right) \longrightarrow \mathrm{H}_{\mathfrak{a}}^{i}(M, N) \longrightarrow \mathrm{H}_{\mathfrak{a}}^{i}\left(M, N / \Gamma_{\mathfrak{a}}(N)\right) \\
& \longrightarrow \operatorname{Ext}_{R}^{i+1}\left(M, \Gamma_{\mathfrak{a}}(N)\right) \longrightarrow \cdots .
\end{aligned}
$$

Since $\mathrm{H}_{\mathfrak{a}}^{0}(M, N)$ has finite length, we have

$$
\operatorname{Supp} \mathrm{H}_{\mathfrak{a}}^{0}(M, N)=\operatorname{Ass}_{\operatorname{Hom}_{R}}\left(M, \Gamma_{\mathfrak{a}}(N)\right)=\operatorname{Ass} \Gamma_{\mathfrak{a}}(N) ;
$$

so that $\Gamma_{\mathfrak{a}}(N) \in \mathcal{S}$. Thus $\operatorname{Ext}_{R}^{i}\left(M, \Gamma_{\mathfrak{a}}(N)\right) \in \mathcal{S}$ for all $i \in \mathbb{N}_{0}$. It follows that for each $i \in \mathbb{N}_{0}, \mathrm{H}_{\mathfrak{a}}^{i}(M, N) \in \mathcal{S}$ if and only if $\mathrm{H}_{\mathfrak{a}}^{i}\left(M, N / \Gamma_{\mathfrak{a}}(N)\right) \in \mathcal{S}$. Also we have $\mathrm{H}_{\mathfrak{a}}^{i}(M, N)$ is Artinian if and only if $\mathrm{H}_{\mathfrak{a}}^{i}\left(M, N / \Gamma_{\mathfrak{a}}(N)\right)$ is Artinian. Hence we can replace $N$ by $N / \Gamma_{\mathfrak{a}}(N)$ and assume that $N$ is an $\mathfrak{a}$-torsion free $R$-module. Thus there exists an element $x \in \mathfrak{a}$ which is a non-zero divisor on $N$. The exact sequence

$$
0 \longrightarrow N \stackrel{x}{\longrightarrow} N \longrightarrow N / x N \longrightarrow 0
$$

induces the long exact sequence

$$
\cdots \longrightarrow \mathrm{H}_{\mathfrak{a}}^{i}(M, N) \stackrel{x}{\longrightarrow} \mathrm{H}_{\mathfrak{a}}^{i}(M, N) \stackrel{f_{i}}{\longrightarrow} \mathrm{H}_{\mathfrak{a}}^{i}(M, N / x N) \longrightarrow \mathrm{H}_{R}^{i+1}(M, N) \longrightarrow \cdots .
$$

Since $\mathrm{H}_{\mathfrak{a}}^{i}(M, N)$ is Artinian for all $i<r$, we may use the above sequence to see that $\mathrm{H}_{\mathfrak{a}}^{i}(M, N / x N)$ is Artinian for all $i<r-1$. On the other hand, $\mathrm{H}_{\mathfrak{a}}^{r}(M, N)$ is not Artinian. Hence, using the above exact sequence and [5, Theorem 7.1.2], we see that $0:_{\mathrm{H}_{\mathfrak{a}}^{r}(M, N)} x \cong \mathrm{H}_{\mathfrak{a}}^{r-1}(M, N / x N) / \operatorname{im} f_{r-1}$ is not Artinian. Thus $\mathrm{H}_{\mathfrak{a}}^{r-1}(M, N / x N)$ is not Artinian; and hence, by inductive hypothesis, $\mathrm{H}_{\mathfrak{a}}^{r-1}(M, N / x N) \notin \mathcal{S}$. So, again 
by using the above sequence, we get $\mathrm{H}_{\mathfrak{a}}^{r}(M, N) \notin \mathcal{S}$. This completes the inductive step.

Corollary 4.3. Suppose that $\operatorname{Supp} L=\operatorname{Supp} M / \mathfrak{a} M$. Then

$$
\inf \left\{i \in \mathbb{N}_{0} \mid \mathrm{H}_{\mathfrak{a}}^{i}(M, N) \text { is not Artinian }\right\}=\inf \left\{i \in \mathbb{N}_{0} \mid \operatorname{dim} \operatorname{Ext}_{R}^{i}(L, N)>0\right\} .
$$

Proof. Let $n \in \mathbb{N}_{0}$. Then, by the Theorem 4.2 $\mathrm{H}_{\mathfrak{a}}^{i}(M, N)$ is an Artinian $R$ module for all $i \leq n$ if and only if $n<\mathrm{f}-\operatorname{grad}\left(\mathfrak{m}_{1} \cap \ldots \cap \mathfrak{m}_{t}, \mathfrak{a}+\operatorname{Ann} M, N\right)$ for some maximal ideals $\mathfrak{m}_{1}, \ldots, \mathfrak{m}_{t}$ of $R$. By the Remarks 2.4 (i), it is equivalent to $\operatorname{Supp} \operatorname{Ext}_{R}^{i}(L, N) \subseteq\left\{\mathfrak{m}_{1}, \ldots, \mathfrak{m}_{t}\right\}$ for some maximal ideals $\mathfrak{m}_{1}, \ldots, \mathfrak{m}_{t}$ of $R$ and for all $i \leq n$. This proves the assertion.

The following corollary extend the main result of [25] to the generalized local cohomology modules.

Corollary 4.4. Let $n \in \mathbb{N}$. Then $\mathrm{H}_{\mathfrak{a}}^{i}(M, N)$ is Artinian for all $i<n$ if and only if $\mathrm{H}_{\mathfrak{a} R_{\mathfrak{p}}}^{i}\left(M_{\mathfrak{p}}, N_{\mathfrak{p}}\right)$ is Artinian for all $i<n$ and all prime ideal $\mathfrak{p}$.

Proof. This is immediate by the Corollary 4.3 .

Corollary 4.5. Let $\bar{R}=R /(\mathfrak{a}+\operatorname{Ann} M+\operatorname{Ann} N)$ be a semi local ring and let $\mathfrak{r}$ be the inverse image of the Jacobson radical of $\bar{R}$ in $R$. Then we have

$$
\begin{aligned}
\mathrm{f}-\operatorname{grad}(\mathfrak{r}, \mathfrak{a}+\operatorname{Ann} M, N) & =\inf \left\{i \in \mathbb{N}_{0} \mid \mathrm{H}_{\mathfrak{a}}^{i}(M, N) \text { is not Artinian }\right\} \\
& =\inf \left\{i \in \mathbb{N}_{0} \mid \mathrm{H}_{\mathfrak{a}}^{i}(M, N) \nsucceq \mathrm{H}_{\mathfrak{r}}^{i}(M, N)\right\}
\end{aligned}
$$

Proof. The first equality is immediate by Theorem 4.2. To prove the second equality, let $n \leq \mathrm{f}-\operatorname{grad}(\mathfrak{r}, \mathfrak{a}+\operatorname{Ann} M, N)$ and let $x_{1}, \ldots, x_{n}$ be an $\mathfrak{r}$-filter regular $N$ sequence in $\mathfrak{a}+\operatorname{Ann} M$. Then $x_{1}, \ldots, x_{n}$ is an $\mathfrak{a}+\operatorname{Ann} M$-filter regular $N$-sequence. So by Theorem 3.1(i),

$$
\mathrm{H}_{\mathfrak{a}}^{i}(M, N) \cong \mathrm{H}_{\mathfrak{a}+\operatorname{Ann} M}^{i}(M, N) \cong \mathrm{H}_{\left(x_{1}, \ldots, x_{n}\right)}^{i}(M, N) \cong \mathrm{H}_{\mathfrak{r}}^{i}(M, N)
$$

for all $i<n$. If $\mathrm{f}-\operatorname{grad}(\mathfrak{r}, \mathfrak{a}+\operatorname{Ann} M, N)=\infty$, then the above argument shows that, $\inf \left\{i \in \mathbb{N}_{0} \mid \mathrm{H}_{\mathfrak{a}}^{i}(M, N) \nsucceq \mathrm{H}_{\mathfrak{r}}^{i}(M, N)\right\}=\infty$ and therefore the required equality holds. Therefore, we may assume that $\mathrm{f}-\operatorname{grad}(\mathfrak{r}, \mathfrak{a}+\operatorname{Ann} M, N)=n<\infty$. By the first equality, $\mathrm{H}_{\mathfrak{a}}^{n}(M, N)$ is not Artinian while $\mathrm{H}_{\mathfrak{r}}^{n}(M, N)$ is Artinian. Hence the second equality holds.

It was shown in [28, Theorem 2.2] that if $\operatorname{dim} R / \mathfrak{a}=0$, then $\mathrm{H}_{\mathfrak{a}}^{i}(M, N)$ is Artinian for all $i \in \mathbb{N}_{0}$. The following corollary is a generalization of this.

Corollary 4.6. Let $\bar{R}=R /(\mathfrak{a}+\operatorname{Ann} M+\operatorname{Ann} N)$. Then $\mathrm{H}_{\mathfrak{a}}^{i}(M, N)$ is an Artinian $R$-module for all $i \in \mathbb{N}_{0}$ if and only if $\operatorname{dim} \bar{R}=0$. In particular, $\operatorname{Ext}_{R}^{i}(M, N)$ has finite length for all $i \in \mathbb{N}_{0}$ if and only if $\operatorname{dim} R /(\operatorname{Ann} M+\operatorname{Ann} N)=0$.

Proof. Assume that $\mathfrak{p}$ is a prime ideal of $R$. By the Corollary 4.5, $\mathrm{H}_{\mathfrak{a} R_{\mathfrak{p}}}^{i}\left(M_{\mathfrak{p}}, N_{\mathfrak{p}}\right)$ is Artinian for all $i<n$ if and only if f-depth $\left((\mathfrak{a}+\operatorname{Ann} M) R_{\mathfrak{p}}, N_{\mathfrak{p}}\right)=\infty$ or equivalently $\operatorname{dim}_{R_{\mathfrak{p}}} N_{\mathfrak{p}} /\left(\mathfrak{a} R_{\mathfrak{p}}+(\operatorname{Ann} M) R_{\mathfrak{p}}\right) N_{\mathfrak{p}}=0$ (Remark 2.3) . Now, the result follows by corollary 4.4 


\section{Attached PRimes of the top Generalized local COHOMOlOGy MODULES}

Let $X \neq 0$ be an $R$-module. If, for every $x \in R$, the endomorphism on $X$ given by multiplication by $x$ is either nilpotent or surjective, then $\mathfrak{p}=\sqrt{\operatorname{Ann} X}$ is prime and $X$ is called a $\mathfrak{p}$-secondary $R$-module. If for some secondary submodules $X_{1}, \ldots, X_{n}$ of $X$ we have $X=X_{1}+\ldots+X_{n}$, then we say that $X$ has a secondary representation. One may assume that the prime ideals $\mathfrak{p}_{i}=\sqrt{\operatorname{Ann} X_{i}}, i=1, \ldots, n$, are distinct and, by omitting redundant summands, that the representation is minimal. Then the set Att $X=\left\{\mathfrak{p}_{1}, \ldots, \mathfrak{p}_{n}\right\}$ does not depend on the choice of a minimal secondary representation of $X$. Every element of Att $X$ is called an attached prime ideal of $X$. It is well known that an Artinian $R$-module has a secondary representation. The reader is referred to [14] for more information about the theory of secondary representation.

Let $(R, \mathfrak{m})$ be a local ring and $n=\operatorname{dim} N<\infty$ and $d=\operatorname{proj} \operatorname{dim} M<\infty$. It was proved in [9, Theorem 2.3] that $\mathrm{H}_{\mathfrak{a}}^{n+d}(M, N)$ is Artinian and that

$$
\operatorname{Att}_{\mathfrak{a}}^{n+d}(M, N)=\left\{\mathfrak{p} \in \operatorname{Ass} N \mid \operatorname{cd}_{\mathfrak{a}}(M, R / \mathfrak{p})=n+d\right\},
$$

where, for an $R$-module $Y, \operatorname{cd}_{\mathfrak{a}}(M, Y)$ is the greatest integer $i$ such that $\mathrm{H}_{\mathfrak{a}}^{i}(M, Y) \neq$ 0 . Notice that $\operatorname{cd}_{\mathfrak{a}}(M, N) \leq d+n$ [3. Lemma 5.1]. Next, we prove the above result without the local assumption on $R$. The following lemmas are needed.

Lemma 5.1 ( [1] Theorem A and B ). Let $\operatorname{proj} \operatorname{dim} M<\infty$. Then

(i) $\operatorname{cd}_{\mathfrak{a}}(M, N) \leq \operatorname{cd}_{\mathfrak{a}}(M, L)$ whenever $\operatorname{Supp} N \subseteq \operatorname{Supp} L$.

(ii) $\operatorname{cd}_{\mathfrak{a}}(M, L)=\max \left\{\operatorname{cd}_{\mathfrak{a}}(M, N), \operatorname{cd}_{\mathfrak{a}}(M, K)\right\}$ whenever $0 \rightarrow N \rightarrow L \rightarrow K \rightarrow 0$ is an exact sequence.

Lemma 5.2. Let proj $\operatorname{dim} M<\infty, \operatorname{dim} N<\infty, t=\operatorname{cd}_{\mathfrak{a}}(M, N) \geq 0$ and

$$
\Sigma=\left\{L \varsubsetneqq N \mid \operatorname{cd}_{\mathfrak{a}}(M, L)<t\right\} .
$$

Then $\Sigma$ has the largest element with respect to inclusion, L say, and the following statements hold.

(i) If $K$ is a non-zero submodule of $N / L$, then $\operatorname{cd}_{\mathfrak{a}}(M, K)=t$.

(ii) $\mathrm{H}_{\mathfrak{a}}^{t}(M, N) \cong \mathrm{H}_{\mathfrak{a}}^{t}(M, N / L)$.

(iii) If $t=\operatorname{proj} \operatorname{dim} M+\operatorname{dim} N$, then

$$
\operatorname{Ass} N / L=\left\{\mathfrak{p} \in \operatorname{Ass} N \mid \operatorname{cd}_{\mathfrak{a}}(M, R / \mathfrak{p})=t\right\} .
$$

Proof. Since $N$ is Noetherian, $\Sigma$ has a maximal element, say $L$. Now assume that $L_{1}, L_{2}$ are elements of $\Sigma$. Using the exact sequence

$$
0 \rightarrow L_{1} \cap L_{2} \rightarrow L_{1} \oplus L_{2} \rightarrow L_{1}+L_{2} \rightarrow 0
$$

and Lemma 5.1 we see that $t>\operatorname{cd}_{\mathfrak{a}}\left(M, L_{1}+L_{2}\right)$. Hence the sum of any two elements of $\Sigma$ is again in $\Sigma$. It follows that $L$ contains every element of $\Sigma$; and so it is the largest one.

(i) Let $K=K^{\prime} / L$ be a non-zero submodule of $N / L$. Since $L$ is the largest element of $\Sigma$, by applying Lemma 5.1 to the exact sequence

$$
0 \rightarrow L \rightarrow K^{\prime} \rightarrow K \rightarrow 0
$$

we see that $t=\operatorname{cd}_{\mathfrak{a}}(M, K)$.

(ii) The exact sequence $0 \rightarrow L \rightarrow N \rightarrow N / L \rightarrow 0$ induces the exact sequence

$$
0=\mathrm{H}_{\mathfrak{a}}^{t}(M, L) \rightarrow \mathrm{H}_{\mathfrak{a}}^{t}(M, N) \rightarrow \mathrm{H}_{\mathfrak{a}}^{t}(M, N / L) \rightarrow \mathrm{H}_{\mathfrak{a}}^{t+1}(M, L)=0 .
$$


Thus $\mathrm{H}_{\mathfrak{a}}^{t}(M, N) \cong \mathrm{H}_{\mathfrak{a}}^{t}(M, N / L)$.

(iii) Assume that $\operatorname{cd}_{\mathfrak{a}}(M, N)=\operatorname{proj} \operatorname{dim} M+\operatorname{dim} N$. For each $\mathfrak{p}$ in $\operatorname{Ass} L$, we have $\operatorname{cd}_{\mathfrak{a}}(M, R / \mathfrak{p})<t$; so that

$$
\left\{\mathfrak{p} \in \operatorname{Ass} N \mid \operatorname{cd}_{\mathfrak{a}}(M, R / \mathfrak{p})=t\right\} \subseteq \operatorname{Ass} N / L .
$$

To establish the reverse inclusion, let $\mathfrak{p} \in \operatorname{Ass} N / L$. Then by (i) and [3, Lemma 5.1] $t=\operatorname{proj} \operatorname{dim} M+\operatorname{dim} R / \mathfrak{p}$. Therefore $\mathfrak{p} \in \operatorname{Ass} N$ and equality holds.

Theorem 5.3. Let $d=\operatorname{proj} \operatorname{dim} M<\infty$ and $n=\operatorname{dim} N<\infty$. Then the $R$-module $\mathrm{H}_{\mathfrak{a}}^{n+d}(M, N)$ is Artinian and

$$
\operatorname{Att}_{\mathfrak{a}}^{n+d}(M, N)=\left\{\mathfrak{p} \in \operatorname{Ass} N \mid \operatorname{cd}_{\mathfrak{a}}(M, R / \mathfrak{p})=n+d\right\} .
$$

Proof. Let $\boldsymbol{x}=x_{1}, \ldots, x_{n}$ be an $\mathfrak{a}$-filter regular $N$-sequence in $\mathfrak{a}$ and let $E^{\bullet}$ be the minimal injective resolution of $\mathrm{H}_{(\boldsymbol{x})}^{n}(N)$. Since, by [5, Exercise 7.1.7], $\mathrm{H}_{(\boldsymbol{x})}^{n}(N)$ is Artinian, every component of $E^{\bullet}$ is Artinian. On the other hand by 3.1

$$
\mathrm{H}_{\mathfrak{a}}^{n+d}(M, N) \cong \mathrm{H}_{\mathfrak{a}}^{d}\left(M, \mathrm{H}_{(\boldsymbol{x})}^{n}(N)\right) \cong H^{d}\left(\operatorname{Hom}_{R}\left(M, \Gamma_{\mathfrak{a}}\left(E^{\bullet}\right)\right)\right) .
$$

It follows that $\mathrm{H}_{\mathfrak{a}}^{n+d}(M, N)$ is Artinian.

Now we prove that $\operatorname{Att}_{\mathfrak{a}}^{n+d}(M, N)=\left\{\mathfrak{p} \in \operatorname{Ass} N \mid \operatorname{cd}_{\mathfrak{a}}(M, R / \mathfrak{p})=n+d\right\}$. If $\operatorname{cd}_{\mathfrak{a}}(M, N)<n+d$, then $\operatorname{Att}_{\mathfrak{a}}^{n+d}(M, N)=\emptyset=\left\{\mathfrak{p} \in \operatorname{Ass} N \mid \operatorname{cd}_{\mathfrak{a}}(M, R / \mathfrak{p})=n+d\right\}$. So one can assume that $t=\operatorname{cd}_{\mathfrak{a}}(M, N)=n+d$. Let $L$ be the largest submodule of $N$ such that $\operatorname{cd}_{\mathfrak{a}}(M, L)<t$. By Lemma 5.2 there is no non-zero submodule $K$ of $N / L$ such that $\operatorname{cd}_{\mathfrak{a}}(M, K)<t$. Also we have $\mathrm{H}_{\mathfrak{a}}^{t}(M, N) \cong$ $\mathrm{H}_{\mathfrak{a}}^{t}(M, N / L)$ and $\operatorname{Ass} N / L=\left\{\mathfrak{p} \in \operatorname{Ass} N \mid \operatorname{cd}_{\mathfrak{a}}(M, R / \mathfrak{p})=t\right\}$. Moreover $t=$ $\operatorname{cd}_{\mathfrak{a}}(M, N / L)=\operatorname{proj} \operatorname{dim} M+\operatorname{dim} N / L$. Thus we may replace $N$ by $N / L$ and prove that $\operatorname{Att}_{\mathfrak{a}}^{t}(M, N)=\operatorname{Ass} N$. Now, for any non-zero submodule $K$ of $N$, $\operatorname{cd}_{\mathfrak{a}}(M, K)=t$ and $\operatorname{dim} K=n$.

Assume that $\mathfrak{p} \in \operatorname{Att}_{\mathfrak{a}}^{t}(M, N)$. We have $\mathfrak{p} \supseteq \operatorname{Ann}_{\mathfrak{a}}^{t}(M, N) \supseteq \operatorname{Ann} N$. Hence $\mathfrak{p} \in \operatorname{Supp} N$. Now Let $x \in R \backslash \bigcup_{\mathfrak{p} \in \operatorname{Ass} N} \mathfrak{p}$. The exact sequence

$$
0 \longrightarrow N \stackrel{x}{\longrightarrow} N \longrightarrow N / x N \longrightarrow 0
$$

induces the exact sequence

$$
\mathrm{H}_{\mathfrak{a}}^{t}(M, N) \stackrel{x}{\rightarrow} \mathrm{H}_{\mathfrak{a}}^{t}(M, N) \rightarrow \mathrm{H}_{\mathfrak{a}}^{t}(M, N / x N)=0 .
$$

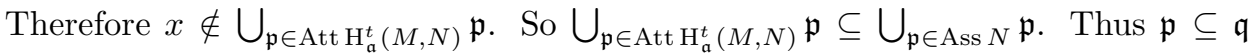
for some $\mathfrak{q} \in \operatorname{Ass} N$. Hence $\mathfrak{p}=\mathfrak{q}$ and $\operatorname{Att}_{\mathfrak{a}}^{t}(M, N) \subseteq \operatorname{Ass} N$. Next we prove the reverse inclusion. Let $\mathfrak{p} \in \operatorname{Ass} N$ and let $T$ be a $\mathfrak{p}$-primary submodule of $N$. We have $t=\operatorname{cd}_{\mathfrak{a}}(M, R / \mathfrak{p})=\operatorname{cd}_{\mathfrak{a}}(M, N / T)$. Moreover $N / T$ has no non-zero submodule $K$ such that $\operatorname{cd}_{\mathfrak{a}}(M, K)<t$. Hence, using the above argument, one can show that Att $\mathrm{H}_{\mathfrak{a}}^{t}(M, N / T) \subseteq \operatorname{Ass} N / T=\{\mathfrak{p}\}$. It follows that

$$
\{\mathfrak{p}\}=\operatorname{Att}_{\mathfrak{a}}^{t}(M, N / T) \subseteq \operatorname{Att}_{\mathfrak{a}}^{t}(M, N) .
$$

This completes the proof.

Corollary 5.4. Let $d=\operatorname{proj} \operatorname{dim} M<\infty$ and $n=\operatorname{dim} N<\infty$. Then

$$
\operatorname{Att}_{\mathfrak{a}}^{n+d}(M, N) \subseteq \operatorname{Supp} M \cap \operatorname{Att}_{\mathfrak{a}}^{n}(N) .
$$


Proof. If $\operatorname{Att}_{\mathfrak{a}}^{n+d}(M, N)=\emptyset$, there is nothing to prove. Assume that $\mathfrak{p} \in$ $\operatorname{Att}_{\mathfrak{a}}^{n+d}(M, N)$. Then, by 5.3, $\mathfrak{p} \in \operatorname{Ass} N$ and $\mathrm{H}_{\mathfrak{a}}^{n+d}(M, R / \mathfrak{p}) \neq 0$. Next one can use the spectral sequence

$$
E_{2}^{p, q}=\operatorname{Ext}_{R}^{p}\left(M, \mathrm{H}_{\mathfrak{a}}^{q}(R / \mathfrak{p})\right) \underset{p}{\Longrightarrow} \mathrm{H}_{\mathfrak{a}}^{p+q}(M, R / \mathfrak{p})
$$

to see that $\mathrm{H}_{\mathfrak{a}}^{n+d}(M, R / \mathfrak{p}) \cong \operatorname{Ext}_{R}^{d}\left(M, \mathrm{H}_{\mathfrak{a}}^{n}(R / \mathfrak{p})\right)$. Therefore $\mathrm{H}_{\mathfrak{a}}^{n}(R / \mathfrak{p}) \neq 0$; and hence $\operatorname{cd}_{\mathfrak{a}}(R / \mathfrak{p})=n$. Thus, again by $5.3, \mathfrak{p} \in \operatorname{Att}_{\mathfrak{a}}^{n}(N)$. Also, we have $\mathfrak{p} \supseteq$ $\operatorname{Ann} \operatorname{Ext}_{R}^{d}\left(M, \mathrm{H}_{\mathfrak{a}}^{n}(N)\right) \supseteq \operatorname{Ann} M$, which completes the proof.

Let $X$ be an $R$-module. Set $E=\bigoplus_{\mathfrak{m} \in \max R} E(R / \mathfrak{m})$ (minimal injective cogenerator of $R$ ) and $D=\operatorname{Hom}_{R}(\cdot, E)$. We note that the canonical map $X \rightarrow D D X$ is an injection. If this map is an isomorphism we say that $X$ is (Matlis) reflexive. The following lemma yields a classification of modules which are reflexive with respect to $E$.

Lemma 5.5 (2 Theorem 12). An R-module $X$ is reflexive if and only if it has a finite submodule $S$ such that $X / S$ is artinian and that $R / A n n X$ is a complete semilocal ring.

Assume that $\mathfrak{a} \subseteq \mathfrak{b}$ and $R / \mathfrak{a}$ is a complete semilocal ring. By above lemma $R / \mathfrak{a}$ is reflexive as an $R$-module. On the other hand, the category of reflexive $R$-modules is a Serre subcategory of the category of $R$-modules. Therefore $R / \mathfrak{b}$ is reflexive as an $R$-module and hence, by the above lemma, $R / \mathfrak{b}$ is a complete semilocal ring. We shall use the conclusion of this discussion in the proof of the next theorem.

Theorem 5.6. Let $M, N$ be two finite $R$-modules with $\operatorname{proj} \operatorname{dim} M=d<\infty$ and $\operatorname{dim} N=n<\infty$. Let $\mathfrak{b}=$ Ann $\mathrm{H}_{\mathfrak{a}}^{n}(N)$. If $R / \mathfrak{b}$ is a complete semilocal ring, then

$$
\operatorname{Att}_{\mathfrak{a}}^{n+d}(M, N)=\operatorname{Supp} \operatorname{Ext}_{R}^{d}(M, R) \cap \operatorname{Att}_{\mathfrak{a}}^{n}(N) .
$$

In particular, if in addition, $\operatorname{proj} \operatorname{dim}_{R_{\mathfrak{p}}} M_{\mathfrak{p}}=\operatorname{proj} \operatorname{dim} M$ for all $\mathfrak{p} \in \operatorname{Supp} M$, then

$$
\operatorname{Att}_{\mathfrak{a}}^{n+d}(M, N)=\operatorname{Supp} M \cap \operatorname{Att}_{\mathfrak{a}}^{n}(N) .
$$

Proof. Since $\operatorname{Ext}_{R}^{d}(M, \cdot)$ is a right exact $R$-linear covariant functor,we have

$$
\mathrm{H}_{\mathfrak{a}}^{n+d}(M, N) \cong \operatorname{Ext}_{R}^{d}\left(M, \mathrm{H}_{\mathfrak{a}}^{n}(N)\right) \cong \operatorname{Ext}_{R}^{d}(M, R) \otimes_{R} \mathrm{H}_{\mathfrak{a}}^{n}(N) .
$$

Set $\mathfrak{c}=\operatorname{Ann~}_{\mathfrak{a}}^{n+d}(M, N)$. It is clear that $\mathfrak{b} \subseteq \mathfrak{c}$. Therefore $R / \mathfrak{c}$ is a complete semilocal ring. Now, by Lemma 5.5, [5, Exercise 7.2.10] and [4, VI.1.4 Proposition 10] we have

$$
\begin{aligned}
\operatorname{Att}_{\mathfrak{a}}^{n+d}(M, N) & =\operatorname{Att} D D \mathrm{H}_{\mathfrak{a}}^{n+d}(M, N) \\
& =\operatorname{Ass} D \mathrm{H}_{\mathfrak{a}}^{n+d}(M, N) \\
& =\operatorname{Ass} D\left(\operatorname{Ext}_{R}^{d}(M, R) \otimes_{R} \mathrm{H}_{\mathfrak{a}}^{n}(N)\right) \\
& =\operatorname{Ass} \operatorname{Hom}_{R}\left(\operatorname{Ext}_{R}^{d}(M, R), D \mathrm{H}_{\mathfrak{a}}^{n}(N)\right) \\
& =\operatorname{Supp} \operatorname{Ext}_{R}^{d}(M, R) \cap \operatorname{Ass} D \mathrm{H}_{\mathfrak{a}}^{n}(N) \\
& =\operatorname{Supp} \operatorname{Ext}_{R}^{d}(M, R) \cap \operatorname{Att} D D \mathrm{H}_{\mathfrak{a}}^{n}(N) \\
& =\operatorname{Supp} \operatorname{Ext}_{R}^{d}(M, R) \cap \operatorname{Att} \mathrm{H}_{\mathfrak{a}}^{n}(N)
\end{aligned}
$$

The final assertion follows immediately from the first equality, [18, Lemma 19.1(iii)] and the fact that $\operatorname{Supp} \operatorname{Ext}_{R}^{d}(M, R) \subseteq \operatorname{Supp} M$. 
By Corollary 5.4 Att $\mathrm{H}_{\mathfrak{a}}^{n+d}(M, N) \subseteq \operatorname{Att} \mathrm{H}_{\mathfrak{a}}^{n}(N)$. Next, we give an example to show that this inclusion may be strict even if $(R, \mathfrak{m})$ is a complete regular local ring and $\mathfrak{a}=\mathfrak{m}$. Also, this example shows that the following theorem of Mafi is not true.

[17. Theorem 2.1]: Let $(R, \mathfrak{m})$ be a commutative Notherian local ring and $n=$ $\operatorname{dim} N, d=\operatorname{proj} \operatorname{dim} M<\infty$. If $\mathrm{H}_{\mathfrak{m}}^{n+d}(M, N) \neq 0$, then

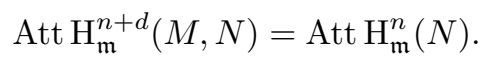

Example 5.7. Let $(R, \mathfrak{m})$ be a complete regular local ring of a dimension $n \geq 2$ and assume that $R$ has two distinct prime ideals $\mathfrak{p}, \mathfrak{q}$ such that $\operatorname{dim} R / \mathfrak{p}=\operatorname{dim} R / \mathfrak{q}=1$. Set $M=R / \mathfrak{p}$ and $N=R / \mathfrak{p} \oplus R / \mathfrak{q}$. Then, by Theorem 5.3,

$$
\operatorname{Att}_{\mathfrak{m}}^{1}(N)=\{\mathfrak{p}, \mathfrak{q}\} .
$$

On the other hand, proj $\operatorname{dim} M=\operatorname{dim} R-\operatorname{depth} M=n-1$ and $\operatorname{dim} N=1$. Now, by Theorem 5.6.

$$
\operatorname{Att} \mathrm{H}_{\mathfrak{m}}^{n}(M, N)=\operatorname{Supp} M \cap \operatorname{Att}_{\mathfrak{m}}^{1}(N)=\{\mathfrak{p}\} .
$$

Therefore [17, Theorem 2.1] is not true. Also, by [5, Proposition 7.2.11],

$$
\sqrt{ }\left(\operatorname{Ann} \mathrm{H}_{\mathfrak{m}}^{n}(M, N)=\bigcap_{\mathfrak{p} \in \operatorname{Att}_{\mathfrak{m}}^{n}(M, N)} \mathfrak{p}=\mathfrak{p}\right.
$$

and

$$
\sqrt{ }\left(\operatorname{Ann} \mathrm{H}_{\mathfrak{m}}^{1}(N)\right)=\bigcap_{\mathfrak{p} \in \operatorname{Att} \mathrm{H}_{\mathfrak{m}}^{1}(N)} \mathfrak{p}=\mathfrak{p} \cap \mathfrak{q} .
$$

Hence, again, Corollary 2.2 and Corollary 2.3 of [17] are not true. We note that, the other results of [17] are concluded from [17, Theorem 2.1, Corollary 2.2 and Corollary 2.3].

It is known that if $(R, \mathfrak{m})$ is a local $\operatorname{ring}$ and $\operatorname{dim} M=n>0$, then $\mathrm{H}_{\mathfrak{m}}^{n}(M)$ is not finite [5, Corollary 7.3.3]. It was proved in [9, Proposition 2.6] that if $d=$ proj $\operatorname{dim} M<\infty$ and $0<n=\operatorname{dim} N$, then $\mathrm{H}_{\mathfrak{m}}^{n+d}(M, N)$ is not finite whenever it is non-zero. Next, we provide a generalization of this result. The following lemma, which is needed in the proof of the next proposition, is elementary.

Lemma 5.8. Let $X$ be an $R$-module. Then $X$ has finite length if and only if $X$ is Artinian and Att $X \subseteq \max R$. Moreover if $X$ has finite length, then Att $X=$ $\operatorname{Supp} X=\operatorname{Ass} X$.

Proposition 5.9. Let $d=\operatorname{proj} \operatorname{dim} M<\infty, 0<n=\operatorname{dim} N<\infty$. If $\mathrm{H}_{\mathfrak{a}}^{n+d}(M, N) \neq$ 0 , then it is not finite.

Proof. Assume that $\mathfrak{p} \in \operatorname{Att}_{\mathfrak{a}}^{n+d}(M, N)$. By 5.3. $\mathrm{H}_{\mathfrak{a}}^{n+d}(M, N)$ is an Artinian $R$ module and $n+d=\operatorname{cd}_{\mathfrak{a}}(M, R / \mathfrak{p})=\operatorname{proj} \operatorname{dim} M+\operatorname{dim} R / \mathfrak{p}$. Therefore $\operatorname{dim} R / \mathfrak{p}=$ $n>0$; So that $\operatorname{Att} \mathrm{H}_{\mathfrak{a}}^{n+d}(M, N) \varsubsetneqq \max R$. It follows that, in view of $5.8, \mathrm{H}_{\mathfrak{a}}^{n+d}(M, N)$ is not finite.

\section{REFERENCES}

[1] J. Amjadi and R. Naghipour, Cohomological dimension of generalized local cohomology modules, Algebra Colloq. 15 (2008), no. 2, 303-308.

[2] R. G. Belshoff, E. E. Enochs and J. R. García Rozas, Generalized Matlis duality, Proc. Amer. Math. Soc. 128 (2000), no. 5, 1307-1312. 
[3] M. H. Bijan-Zadeh, A common generalization of local cohomology theories, Glasgow Math. J. 21 (1980), no. 2, 173-181.

[4] N. Bourbaki, Commutative Algebra, Chapter 1-7, Elements of Mathematics, Springer-Verlage, Berlin, 1998.

[5] M. P. Brodmann and R. Y. Sharp, Local Cohomology: an Algebraic introduction with geometric applications, Cambridge Studies in Advanced Mathematics, 60. Cambridge University Press, Cambridge, 1998.

[6] W. Bruns and J. Herzog, Cohen-Macaulay rings, Cambridge Studies in Advanced Mathematics, 39. Cambridge University Press, Cambridge, 1993.

[7] L. Chu and Z. Tang, On the Artinianness of generalized local cohomology, Comm. Algebra 35 (2007), no. 12, 3821-3827.

[8] M. T. Dibaei and S. Yassemi, Attached primes of the top local cohomology modules with respect to an ideal, Arch. Math. (Basel) 84 (2005), no. 4, 292-297.

[9] Y. Gu and L. Chu, Attached primes of the top generalized local cohomology modules, Bull. Aust. Math. Soc. 79 (2009), no. 1, 59-67.

[10] J. Herzog, Komplexe, Auflösungen und Dualität in der lokalen Algebra, Habilitationsschrift, Universität Regensburg, 1970.

[11] K. Khashyarmanesh and Sh. Salarian, Filter regular sequences and the finiteness of local cohomology modules, Comm. Algebra 26 (1998), no. 8, 2483-2490.

[12] K. Khashyarmanesh, M. Yassi and A. Abbasi, Filter regular sequences and generalized local cohomology modules, Comm. Algebra 32 (2004), no. 1, 253-259.

[13] R. Lü and Z. Tang, The f-depth of an ideal on a module, Proc. Amer. Math. Soc. 130 (2002), no. 7, 1905-1912 (electronic).

[14] I. G. MacDonald, Secondary representation of modules over a commutative ring, Symposia Mathematica, Vol. XI (Convegno di Algebra Commutativa, INDAM, Rome, 1971), pp. 23-43. Academic Press, London, 1973.

[15] I. G. Macdonald and R. Y. Sharp, An elementary proof of the non-vanishing of certain local cohomology modules, Quart. J. Math. Oxford Ser. (2) 23 (1972), 197-204.

[16] A. Mafi, On the associated primes of generalized local cohomology modules, Comm. Algebra 34 (2006), no. 7, 2489-2494.

[17] A. Mafi, Top generalized local cohomology modules, Turkish J. Math. 35 (2011), no. 4, 611-615.

[18] H. Matsumura, Commutative ring theory, Cambridge Studies in Advanced Mathematics, 8. Cambridge University Press, Cambridge, 1986.

[19] L. Melkersson, Some applications of a criterion for Artinianness of a module, J. Pure Appl. Algebra 101 (1995), no. 3, 291-303.

[20] U. Nagel and P. Schenzel, Cohomological annihilators and Castelnuovo-Mumford regularity, Commutative algebra: syzygies, multiplicities, and birational algebra (South Hadley, MA, 1992), 307-328, Contemp. Math., 159, Amer. Math. Soc., Providence, RI, 1994.

[21] J. J. Rotman, An introduction to homological algebra, Pure and Applied Mathematics 85. Academic Press, Inc., New York, 1979.

[22] R. Y. Sharp, Steps in commutative algebra, London Mathematical Society Student Texts, 19. Cambridge University Press, Cambridge, 1990.

[23] J. Stückrad and W. Vogel, Buchsbaum rings and applications. An interaction between algebra, geometry and topology, Springer-Verlag, Berlin, 1986.

[24] N. Suzuki, On the generalized local cohomology and its duality, J. Math. Kyoto Univ. 18 (1978), no. 1, 71-85.

[25] Z. Tang, Local-global principle for the Artinianness of local cohomology modules, Comm. Algebra 40 (2012), no. 1, 58-63.

[26] N. V. Trung, Absolutely superficial sequences, Math. Proc. Cambridge Philos. Soc. 93 (1983), no. $1,35-47$.

[27] S. Yassemi, L. Khatami and T. Sharif, Associated primes of generalized local cohomology modules, Comm. Algebra 30 (2002), no. 1, 327-330.

[28] N. Zamani, On graded generalized local cohomology, Arch. Math. (Basel) 86 (2006), no. 4, $321-330$. 
Department of Mathematics, Science and Research Branch, Islamic Azad University, TEHRAN, IRAN.

E-mail address: alif1387@gmail.com

Department of Mathematics, Science and Research Branch, Islamic Azad University, TEHRAN, IRAN.

E-mail address: tehranian@srbiau.ac.ir

Faculty of Mathematical Sciences and Computer, Tarbiat Moallem University, Tehran, IRAN.

E-mail address: zakeri@tmu.ac.ir 